\title{
Anisotropy of heat capacity in Pauli limited unconventional superconductors *
}

\author{
A. Vorontsov, I. Vekhter \\ Department of Physics and Astronomy, Louisiana State University, Baton Rouge, Louisiana, 70803, USA
}

\begin{abstract}
Diamagnetically coupled magnetic field can be used as a probe of nodal positions in unconventional superconductors. The heat capacity depends on the angle $\phi_{0}$ between the magnetic field and the nodal directions. We show that the anisotropy $C\left(\phi_{0}\right)$ persists even in systems with strong paramagnetic coupling to the electrons's spins.
\end{abstract}

Key words: anisotropic superconductors, heat capacity, vortex, Pauli limit

PACS: 74.20.Rp, 74.25.Bt

Anisotropy of thermodynamic properties of a superconductor in rotated magnetic field is often used to determine the location of the nodes in the superconducting gap at the Fermi surface. Magnetic field serves as a directional probe since the circulating screening supercurrents around vortices excite quasiparticles differently, depending on the orientation of the field with respect to the nodes. While initial work suggested that the density of states and the specific heat have a minimum when the field is applied along a node, we recently argued that the situation is more complex, and minima and maxima interchange in the $T$ - $H$ phase diagram $[1,2]$.

Several superconducting materials, including heavy-fermion $\mathrm{CeCoIn}_{5}$, have strong suppression of superconductivity due to paramagnetic coupling of electron spins to the field. This effect is insensitive to the field orientation and competes with the orbital coupling. We investigate how the anisotropy of the heat capacity is affected by this competition.

We follow the quasiclassical method $[2,3]$ and include interaction of electron spins with the field via Zeeman term. The $4 \times 4$ (spin-up-down-particle-

\footnotetext{
^ Supported by the Board of Regents of Louisiana
}

Preprint submitted to Elsevier Science hole) Green's function $\widehat{g}(\mathbf{R}, \hat{\mathbf{p}} ; \varepsilon)$ satisfies Eilenberger equation[5]

$\left[\varepsilon \widehat{\tau}_{3}-\widehat{v}_{o r b}-\widehat{v}_{Z}-\widehat{\Delta}-\widehat{\sigma}_{i m p}, \widehat{g}\right]+i \mathbf{v}_{f} \cdot \nabla \widehat{g}=0 .(1)$

Here $\widehat{\Delta}(\mathbf{R}, \hat{\mathbf{p}})$ is the mean-field order parameter, $\widehat{\sigma}_{i m p}(\mathbf{R} ; \varepsilon)$ is the impurity self-energy. The orbital coupling of magnetic field is via the vector potential $\mathbf{A}(\mathbf{R})$, while the Zeeman term couples $\mathbf{B}$ to the magnetic moments of electrons $\mu=(g / 2) \mu_{B}$,

$$
\widehat{v}_{o r b}=-\frac{e}{c} \mathbf{v}_{f} \mathbf{A} \widehat{\tau}_{3} \quad \widehat{v}_{Z}=\left(\begin{array}{cc}
\mu \boldsymbol{\sigma} \cdot \mathbf{B} & 0 \\
0 & \mu \boldsymbol{\sigma}^{*} \cdot \mathbf{B}
\end{array}\right) .
$$

In the absence of spin-orbit interaction we choose the direction of the field to be the spin quantization axis. Then all matrices split into independent blocks, for spin up and down. The equations for the two spin directions are independent, and differ only by a spindependent energy shift. For example, the equation for the off-diagonal components of $\widehat{g}(\mathbf{R}, \hat{\mathbf{p}} ; \varepsilon)$ is

$$
\begin{array}{r}
{\left[-2 i(\tilde{\varepsilon} \mp \mu B)+\mathbf{v}_{f}(\hat{\mathbf{p}})\left(\nabla_{\mathbf{R}}-i \frac{2 e}{\hbar c} \mathbf{A}(\mathbf{R})\right)\right] \times} \\
f_{\uparrow, \downarrow}^{R}(\hat{\mathbf{p}}, \mathbf{R} ; \varepsilon)=2 \tilde{\Delta} i g_{\uparrow, \downarrow}^{R}(\hat{\mathbf{p}}, \mathbf{R} ; \varepsilon) .
\end{array}
$$




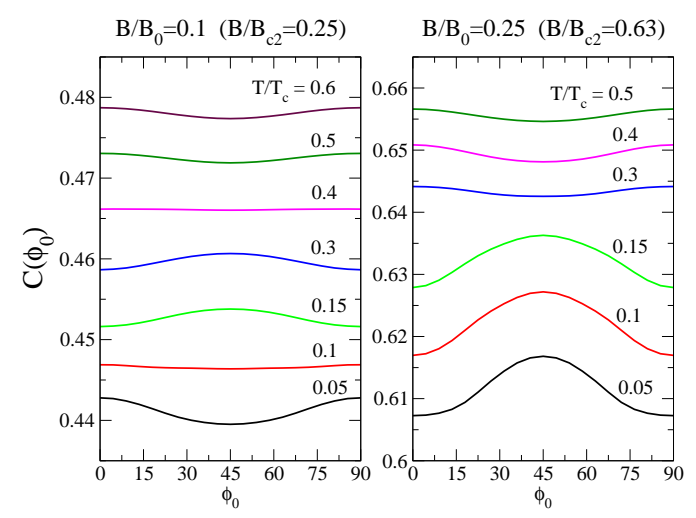

Fig. 1. The heat capacity anisotropy for $Z=0.4$ at different temperatures. Anisotropy curves correspond to points indicated in the right panel of Fig. 2. (The upper curves are shifted down to be on the same scale.)

Here the energy $\tilde{\varepsilon}=\varepsilon-\Sigma_{\uparrow, \downarrow}$ and order parameter $\tilde{\Delta}=\Delta+\Delta_{i m p, \uparrow, \downarrow}$ are renormalized by impurity self-energies. In analogy to Ref.[2], we take the $d_{x^{2}-y^{2}}$ gap $\Delta=\Delta(\mathbf{R}) \sqrt{2} \cos 2 \phi$, with spatial structure given by Abrikosov vortex lattice, and do not consider a possible additional modulation due to Fulde-Ferrell-Larkin-Ovchinnikov state.We solve these equations for Green's function using BrandtPesch-Tewordt approximation.[4] To these we add self-consistency equations on $\widehat{\sigma}_{i m p}$ and $\Delta$. The latter is the only place where two spins add, $\Delta(\mathbf{R}, \hat{\mathbf{p}})=$ $\int \frac{d \varepsilon}{4 \pi i} \tanh \frac{\varepsilon}{2 T} \int \mathrm{d} \hat{\mathbf{p}}^{\prime} V\left(\hat{\mathbf{p}}, \hat{\mathbf{p}}^{\prime}\right) \frac{1}{2}\left(f_{\uparrow}+f_{\downarrow}\right)$.

The specific heat is calculated from the density of states for two spins $N_{\uparrow, \downarrow}$ using,

$C\left(T, \phi_{0}\right)=\int_{-\infty}^{+\infty} d \varepsilon \frac{\varepsilon^{2}\left(N_{\uparrow}\left(T, \phi_{0} ; \varepsilon\right)+N_{\downarrow}\left(T, \phi_{0} ; \varepsilon\right)\right)}{4 T^{2} \cosh ^{2}(\varepsilon / 2 T)}$,

which is valid at low temperature, $T \ll T_{c}$. The calculations are done for a quasi-cylindrical Fermi surface, $p_{f}^{2}=p_{x}^{2}+p_{y}^{2}-r^{2} p_{f}^{2} \cos \left(2 s p_{z} / r^{2} p_{f}\right)$, with parameters $r=s=0.5$ that ensure the $3 \mathrm{D}$ nature of the vortices.[2] Parameter $Z=\mu B_{0} / 2 \pi T_{c}$, where $B_{0}=(c h / 2 e) / 2 \pi \xi_{0}^{2}$, and the in-plane coherence length $\xi_{0}=\hbar v_{f} / 2 \pi T_{c}$, characterises the strength of the Zeeman term.

Figure 1 shows the anisotropy of the specific heat as a function of $T$ and $B$. For $\mathbf{B}$ in the nodal direction, $\phi_{0}=45^{\circ}, C\left(\phi_{0}\right)$ has minima or maxima depending on the temperature and field range. This is similar to the case of purely orbital coupling, $Z=0$, and is due to the interplay between excitation and scattering of quasiparticles by magnetic field, which is affected by the quasiparticles energy $(\sim T)$, and magnitude and the orientation of the field.[2]

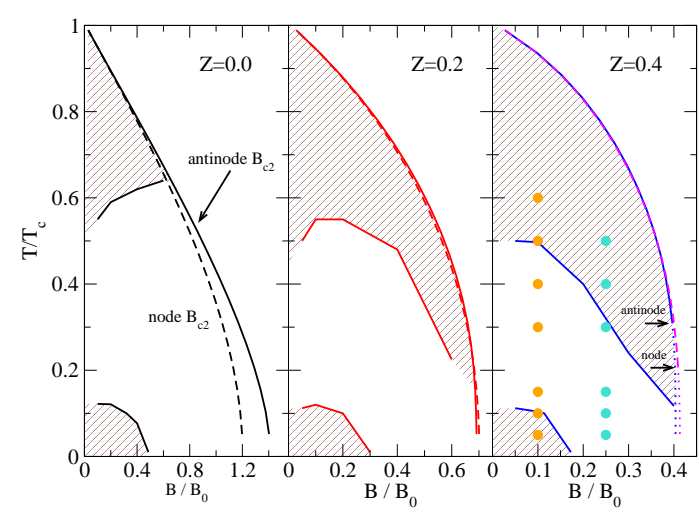

Fig. 2. Phase diagram of the $C\left(T, \phi_{0}\right)$ anisotropy for different strength of the paramagnetic term. In the shaded areas $C\left(\phi_{0}\right)$ has a minimum when the field is along a nodal direction. The arrows in the right panel indicate onset of the first order transition occurring for $Z^{*} \gtrsim 0.35$.

Fig. 2 shows regions of minima and maxima at $\phi_{0}=45^{\circ}$ in the $T-B$ plane, and their evolution with increasing Zeeman term $Z$. Of the two areas where $C\left(\phi_{0}\right)$ has a minimum at $45^{\circ}$, the low- $T$, low$B$ regime is only weakly affected by variation in $Z$. In contrast, the region at high $T$ expands as we increase $Z$, at the expense of the area where a maximum at $\phi_{0}=45^{\circ}$ is observed. Note that the inplane anisotropy of $B_{c 2}$ (present for $Z=0$ ) quickly disappears as we turn on the strength of the Pauli coupling.

The anisotropy of $C\left(\phi_{0}\right)$ is usually measured below $T_{c} / 2[6,7]$, where the effect of the Zeeman coupling is weak. While increased $Z$ expands the high- $T$ "minimum" region, the amplitude of the anisotropy there is probably still below the experimental resolution. We conclude that the Zeeman coupling has weak effect on the observed anisotropy.

\section{References}

[1] I. Vekhter et al., Phys. Rev. B 59 (1999) 9023(R).

[2] A. B. Vorontsov, I. Vekhter, Phys. Rev. Lett. 96 (2006) 237001, and unpublished

[3] A. Houghton, I. Vekhter, Phys. Rev. B 57 (1998) 10831; H. Kusunose, Phys. Rev. B 70 (2004) 054509.

[4] U. Brandt, W. Pesch, L. Tewordt, Z. Phys. 201, 209 (1967);

[5] G. Eilenberger, Z. Phys. 214 (1968) 195; A. I. Larkin, Y. N. Ovchinnikov, Sov. Phys. JETP 28 (1969) 1200.

[6] T. Park et al. , Phys. Rev. Lett. 90 (2003) 177001.

[7] H. Aoki et al. , J. of Physics: Cond. Matt. 16 (2004) L13. 